\title{
Acoustic Experimental Data Analysis of Moving Targets Echoes Observed by Doppler Radars
}

\author{
Milenko Andrić1, ${ }^{*}$ - Boban Bondžulić1 - Bojan Zrnić2 ${ }^{-}$Aleksandar Kari1 - Goran Dikić1 \\ ${ }^{1}$ University of Defence, Military Academy, Belgrade, Serbia \\ ${ }^{2}$ Ministry of Defence, Defence Technology Department, Belgrade, Serbia
}

In this paper we describe the main tasks of ground surveillance radars for security and perimeter protection and targets detection. Our goal is to provide a balanced and comprehensive database to enable reproducible research results in the field of classification of ground moving targets (pattern recognition).

Also, in this paper, we consider received radar echoes data of ground moving targets, and corresponding signals in time - frequency domain using spectrogram and cepstrum. The database, noted as RadEch Database, containing radar echoes from various targets. The objective of the paper is to identify and validate the intrinsic features characterizing the different classes of targets, and subsequently extract salient features for classification

Keywords: cepstrum, classification, Doppler signature, feature extraction, radar echoes database, spectrogram

\section{INTRODUCTION}

The main tasks of ground surveillance radars for defence and specific perimeter protection are detection and classification of ground moving targets. This process usually takes electro magnetic (EM) radars as the base sensors and Doppler effect to estimate radial velocities. EM radars, as sensors, are a well known technology for different surveillance and measurement purposes. Signal processing of EM radars shows some advantages if it transforms into the acoustic audio signals to the end users. Signals from EM radars are very sensitive on the jamming which causes difficulties in terms of its processing, digitalization and final recognizing of their sources.

Acoustic signature as a diagnostic tool has different applications in mechanical engineering [1] and distribution of acoustic waves, its form and characteristic properties initiate different methodologies to estimate [2] behavior and performances of required reflected targets, spare parts, mechanical elements, environmental areas, and components, etc. Due to the above mentioned, in the most applications of ground surveillance radars, moving targets classification is performed using their transformation of EM signals to acoustic in aim to estimate audio-Doppler signature.

The Doppler phenomenon describes the shift in the center frequency of an incident waveform due to the target motion with respect to the radar [3]. Radar produces an audio signal from the Doppler frequency of moving targets. Important classes of ground targets can be distinguished by their audio Doppler signature. While the operator recognizes the moving targets using the audio Doppler signatures by listening an audio channel, this concept leads to unsatisfactory performance, limited by the human operator's senses. In aim to avoid this, miss data base of acoustic signatures transformed from EM radar signals in the loop, is necessary.

To the best of our knowledge, there is only one database with a wide class of target echoes for low resolution surveillance radar. However, there are differences between database description given in [4]. Therefore, extensive experiments with various scenarios were carried out, represented in this paper, in order to obtain such a database (different targets and environments).

The second problem to achieve reliable data about those targets in using audio signals from EM radars is the method of signal processing and recognizing types and states of the targets.

Many current radar-based classification systems employ some type of Doppler or Fourier-based processing, followed by spectrogram and gait analysis to classify detected targets.

In several studies it has been proved that spectrogram-based features could be used for discrimination purposes either between humans and other moving objects or between different persons [5] to [8]. Human spectrograms can be used to reveal information on human behaviour and to determine features about the human target being observed, such as size, gender, action, and speed, too.

Research done in [5] had shown that the human spectrogram is the sum of Doppler shifted signals. Using Short Time Fourier Transform (STFT) and the chirplet transform, they extracted various parameters of the human gait from the signal. Research done in [6], has shown that the radar Doppler signatures 
give detailed information about the movements of the human body parts. The authors focused on the extraction of parameters and described a method for estimating human walking parameters from radar measurements. The application of continuous-wave radar for the detection and classification of people based on their motion has been demonstrated in [7]. Spectral analysis of the output from the radar using a sequence of STFTs was used to extract and to identify some key features of the human walking motion, and to differentiate humans from dogs. Using human gait analysis [8] designed and tested a suicide bomber detection system based on variations in the spectrogram caused by the presence of a bomb.

A target classification algorithms using Doppler signature were presented in [9] to [13]. In [9] a Hidden Markov Model (HMM) classifier was implemented for classification between three classes of targets: personnel, tracked vehicles and wheeled vehicles. A fuzzy logic approach to the automatic classification was presented in [10]. The problem of classification between a walking person, pair of walking persons and slowly moving vehicle was studied in [11]. Time varying velocities and bio-mechanical human locomotion models they used for target classification.

At first glance, cepstrum-based features seem like a promising solution for classification problems. However, the applicability and performances of these features were sometimes not tested in the context of practical systems [12]. Therefore, extensive experiments with various scenarios were carried out in order to obtain a radar echoes database (different targets and environments). In order to identify and validate the main features of the various target classes the STFT and cepstrum analysis are performed.

The basic goal of this paper is presentation of autonomous measured data base and an appropriate method of signal processing for targets recognition, tracked by EM radar, linked with a audio sensor to the end user, as the base for digitalization in a further automatic awareness system.

\section{TARGETS DATABASE DESCRIPTION AND COLLECTION}

The database was obtained using records of short range ground surveillance radar, collected on the memory of computer. This radar is coherent and has from the outset possessed a so called audio output which can be used by the operator to classify targets. When a radio frequency (RF) signal is incident on a target moving towards or away from the radar, the signals reflected from the various components of the target will have a Doppler shift that is proportional to the velocity of those components.

The radar operates in the Ku-band and for this carrier frequency, the Doppler frequencies lie within the audio band, being of the order of a kilohertz, and so can be presented as an audio tone to the radar operator, via headphones. Listened or signed tone was noted that ground moving targets produced a very distinct and characteristic sound that, upon hearing it only a few times, it is easily recognized.

The target sensor, used in database collection, is $16.8 \mathrm{GHz}$ ground surveillance pulse-Doppler radar. The radar parameters are: average power is $5 \mathrm{~mW}$, pulse width $15 \mu \mathrm{s}$, average range resolution $150 \mathrm{~m}$, elevation resolution $7.5^{\circ}$, and azimuth resolution $5^{\circ}$.

For the recording procedure, the target was detected and tracked automatically by the radar, allowing continuous target echo records. The range between the radar and the target was set to be short $(100$ to $1000 \mathrm{~m})$. The moving targets were within the line-of-sight, in the presence of ground clutter with low vegetation and without any interference. The target motions were fully controlled. One target at a time was recorded in each scenario.

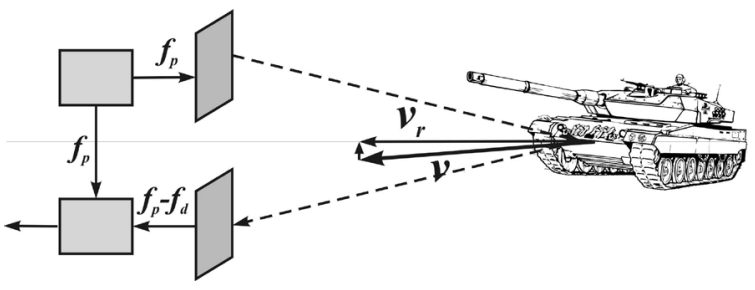

Fig. 1. Principal scheme of Doppler radar

Amplitude of the raw radar data was in the range $\pm 1 \mathrm{~V}$ and the sampling frequency was $4 \mathrm{kHz}$. Audio signal from the radar was connected to laptop sound card microphone input. The radar's baseband audio signal was recorded onto a laptop, where the data was then saved as digitized WAV files. This allowed for the data to be easily processed using MATLAB ${ }^{\mathrm{TM}}$.

The large database of the raw real audio Doppler signals was created through more than 80 different scenarios. The targets were recorded in two different environments. The first environment is the road of 4 $\mathrm{m}$ width, and $800 \mathrm{~m}$ length. The second environment is the rough terrain, with barriers (slews, woods), and with small vegetation. Targets from the following classes were recorded:

1. Person and group of persons - combinations of the following cases were represented in the collected database:

a. Number of persons: 1,3 or more. 
b. Motion: crawling, normal walking, and running.

c. Synchronous / asynchronous motion of persons in a group.

d. Pedestrian, soldier, group of persons, group of soldiers.

e. Go away - from the radar (otherwise toward the radar).

2. Vehicle:

a. Wheeled / Truck vehicle.

b. Speed of motion: normal (20 to $30 \mathrm{~km} / \mathrm{h})$ and fast (30 to $60 \mathrm{~km} / \mathrm{h})$.

3. Vegetation clutter (trees, bush).

The database that was collected during this work is available at [14].

\section{SPECTROGRAM-BASED ANALYSIS OF AUDIO DOPPLER SIGNAL}

Radar target classification has been an active research area. Usually, a feature extraction process plays an essential role for the success of target classification. Due to the highly aspect-dependent nature of the scattered signature, a feature extraction for target classification is an especially difficult problem [10] and [15]. The feature extraction was obtained using a short-time Fast Fourier Transform (FFT).

The most standard approach to analyze a signal with time-varying frequency content is to split the time-domain signal into many segments, and then take the Fourier transform of each segment. This is known as the STFT operation and is defined as:

$$
X_{n}\left(e^{j \omega}\right)=\sum_{m=-\infty}^{\infty} w[n-m] x[m] e^{-j \omega m}
$$

In Eq. (1), $w[n-m]$ is a real window sequence which determines the portion of the input signal that receives emphasis at a particular time index, $n$. The time dependent Fourier transform is clearly a function of two variables: the time index, $n$, which is discrete, and the frequency variable $\omega$, which is continuous.

The magnitude display $\left|X_{n}\left(e^{j \omega}\right)\right|$ is called the spectrogram of the signal. It shows how the frequency spectrum (i.e., one vertical column of the spectrogram) varies as a function of the horizontal time axis. In most applications for classification of radar echoes Doppler signals, spectrogram is an often used solution.

Based on spectral analysis of Doppler signal by spectrogram, central Doppler frequency and width of spectral band around it are possible features for fuzzy variables [11].
The time-frequency analysis of collected signals was performed by a spectrogram. Spectrograms are calculated for the audio Doppler sequences length of 4 seconds (16000 samples).

Spectrogram is represented by $n \times m$ matrix where are:

$$
n=\frac{N F F T}{2}+1
$$

NFFT is the number of FFT points used to calculate the discrete Fourier transform, and:

$$
m=f i x\left(\frac{N_{x}-N O V E R L A P}{L W I N-N O V E R L A P}\right),
$$

where $N_{x}$ is the input sequence length, NOVERLAP the number of samples each segment of input sequence overlaps, LWIN the length of window function, and function fix rounds to the nearest integer towards zero. The Kaiser window function length of 256 and with parameter $\beta=3 \pi$ was applied. Overlapping between adjacent windows was 128 samples (NOVERLAP = 128). Maximum Doppler frequency is equal $2 \mathrm{kHz}$.

Radar target echoes spectrograms of clutter, person walking, person running, walking group, running group, and light wheeled vehicle, are presented in Fig. 2. The Doppler frequency is displayed on the vertical axis and time on the horizontal. The amplitude of the reflected signals grey scale coded with the highest intensity and the lowest. Each target class has unique time-frequency characteristics, which can be used for classification.

The Doppler frequency shift from a target moving with a velocity $v_{r}$ along the line-of-sight of the sensor is:

$$
f_{d}=\frac{2\left|v_{r}\right|}{\lambda},
$$

where $\lambda$ is the sensor wavelength. One limitation of this type of sensor is that it cannot distinguish whether the object is moving towards or away from it, hence there is absolute value.

The clutter signature (Fig. 2a), which is moving vegetation outcome, is located on frequencies below $100 \mathrm{~Hz}$.

An example of Doppler spectrogram from a walking person is presented in Fig. 2b. When humans walk, the motion of various components of the body including the torso, arms, and legs produce a very characteristic Doppler signature.

Human walking motion is quite complex with contributions to these velocity components from each of the upper and lower parts of the extremities. For 


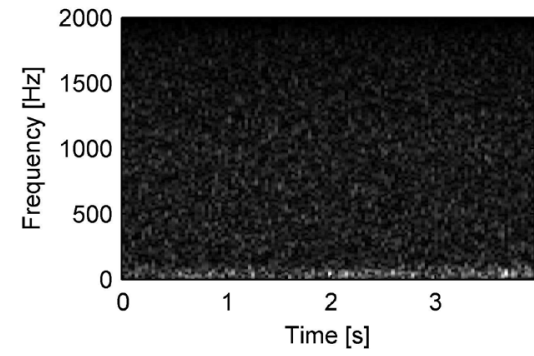

a)

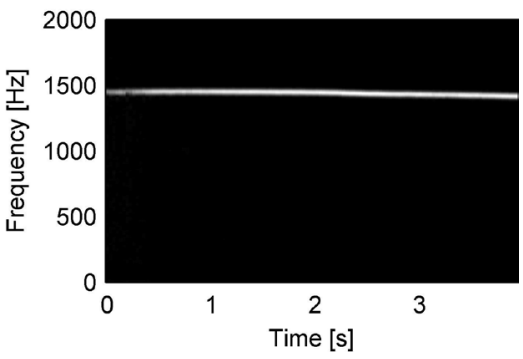

d)

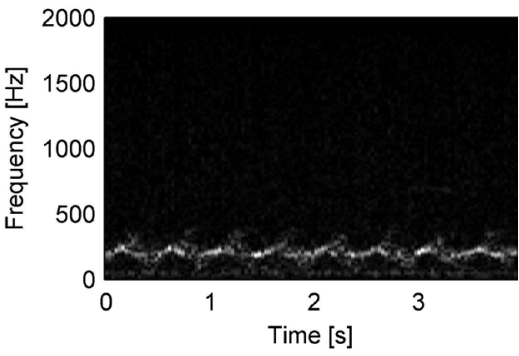

b)

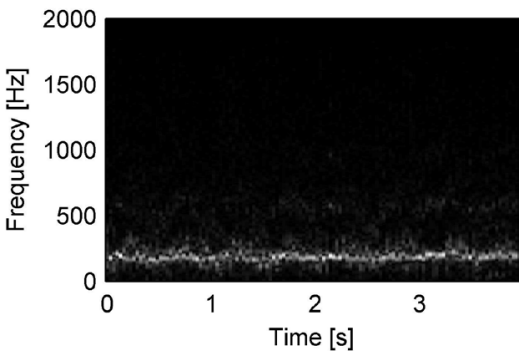

e)

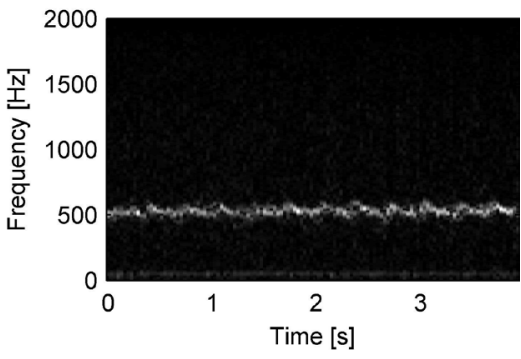

c)

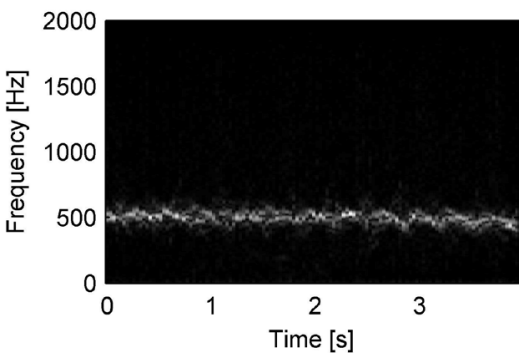

f)

Fig. 2. Spectrograms of radar echo target samples: a) clutter, b) person walking, c) person running, d) vehicle, e) group of persons walking, and f) group of persons running

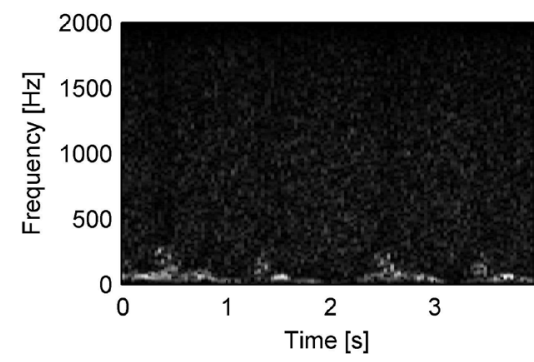

a)

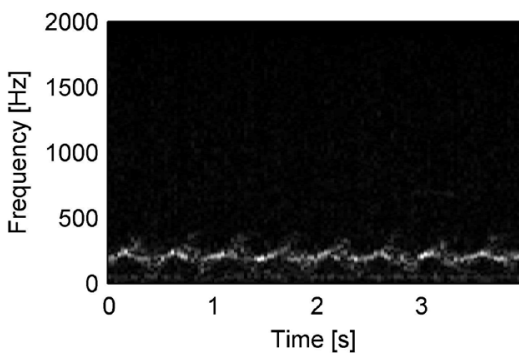

b)

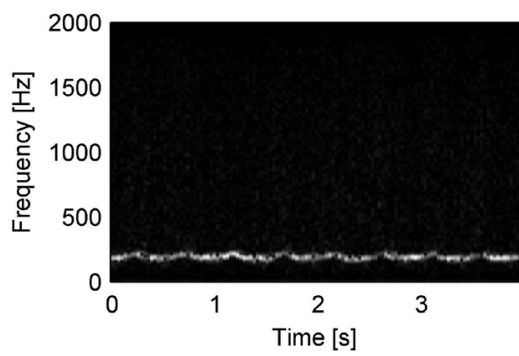

c)

Fig. 3. Spectrograms of a person radar echo samples; a) crawling, b) walking on the road, c) walking on the bush

a person walking with a constant velocity, the signal reflected from the torso, will have a constant Doppler shift. The signals reflected from the swinging legs and arms, will be modulated at the cadence frequency, which is the step or leg swing rate. In general, the arms and legs will have the same periodicity since the arms swing to counterbalance the legs [7].

In Fig. $2 b$ the torso component has a nearly constant Doppler frequency of about $200 \mathrm{~Hz}$ indicating the person was moving with a speed, using Eq. (3), of about $1.75 \mathrm{~m} / \mathrm{s}$. The leg swings introduce a nearly sawtooth modulation around of this torso component.

The dominant contribution to the Doppler signature appears to be the motion of the torso and the legs, the contribution of the arms is not as dominant.

The motion of the torso produces a steady Doppler shifted signal, which is modulated by the motion of the swinging arms and legs that results in a Doppler signature which is very characteristic of humans.

The envelope of the curve corresponds to the motions of the legs and arms, which have smaller cross sections than the torso, so they are less detectable at a distance.

Doppler spectrogram from a running man is presented in Fig. 2c. There is a specific man signature which is characterized by the low Doppler frequency and with a component which oscillates in frequency (characteristic quasi-periodic signal).

A walking person Doppler signature usually lies from 100 to $300 \mathrm{~Hz}$, and a running person Doppler shift lies from 350 to $600 \mathrm{~Hz}$.

The comparative analysis of spectral characteristics of these two target classes shows a 
difference in central Doppler frequency and the width of the spectral line around central Doppler frequency. As the speed of man increases, central Doppler frequency and the appropriate spectral band width also increase.

As the number of persons in a group increases, irrespective of motion type, the spectral band widths also increase (Figs. 2e and f).

A spectrogram from light-wheeled vehicle (car) is seen in Fig. 2d. The signature from moving wheeled vehicle has one dominant spectral line (Doppler frequency) and narrow band of spectral components around central Doppler frequency because the wheeled vehicle is a compact target without moving subreflectors. Tist is the reason for the sound like a pure tone in the operator's headphones. The target has a nearly constant Doppler frequency of about $1400 \mathrm{~Hz}$ indicating the car was moving with a speed, using Eq. (3), of about $12.5 \mathrm{~m} / \mathrm{s}(45 \mathrm{~km} / \mathrm{h})$.

As a conclusion, the band of spectral components around central Doppler frequency is the smallest in the case of a wheeled vehicle in comparison with the walking and running man case. Consequently, in the case when central Doppler frequencies of a running man and a wheeled vehicle with low velocities are similar, they may be used to resolve this type of classification conflict the width of spectral band around the central Doppler frequencies.

Target signature may significantly vary from one scenario, which is illustrated in Fig. 3, where are spectrograms of a crawling person, a person walking on the road, and a person walking in the bush, are shown.

A crawling person spectrogram, (Fig. 3a), is periodic, but with pauses, due to type of motion.

In Figs. $3 \mathrm{~b}$ and $\mathrm{c}$ the torso component has a nearly constant Doppler frequency of about $200 \mathrm{~Hz}$. Audio echoes are modulated with ambient conditions. The leg swings are hidden with vegetation in Fig. 3c.

\section{CEPSTRUM-BASED ANALYSIS OF RADAR ECHOES}

A cepstrum is the result of taking the inverse Fourier transform (FT) of the log spectrum as if it were a signal. Its name was derived by reversing the first four letters of spectrum. There is a complex cepstrum, a real cepstrum, a power cepstrum, and phase cepstrum.

The cepstrum $c[n]$ of a discrete-time signal $x[n]$ is defined as [12] and [13]:

$$
c[n]=F^{-1}\{\log (F\{x[n]\})\},
$$

where $F[\times]$, and $F^{-1}[\times]$ are the Discrete Fourier and the inverse Fourier transforms, respectively.

In Fig. 4 are shown first sixteen significant real cepstrum coefficients for same sequences used in spectrogram analysis. Values of the rest of the coefficients are very small and they are not showed. Generally, cepstrum concentrate energy in few cepstrum coefficients values.

In this paper we analyzed a dependence of cepstrum coefficients from central Doppler frequency and spectral width around it throught two experiments.

In first experiment we analyzed a dependence of cepstrum coefficients from central Doppler frequency. For this purpose we used 20 sequences from each of three major group of moving targets (one person, group of persons and vehicle). All these sequences we grouped in three classes based on central Doppler frequency: below $300 \mathrm{~Hz}$, between 500 and $700 \mathrm{~Hz}$ and above $1400 \mathrm{~Hz}$.

We varied sequence duration from 0.125 to 2 s. Number of points used to calculate the Discrete Fourier transform is 1024.

Cepstrum coefficients are widely used in speech and speaker recognition applications and we applied them to the radar data. For the illustration purposes, samples of the second and the third cepstrum coefficients are analysed in Fig. 5.

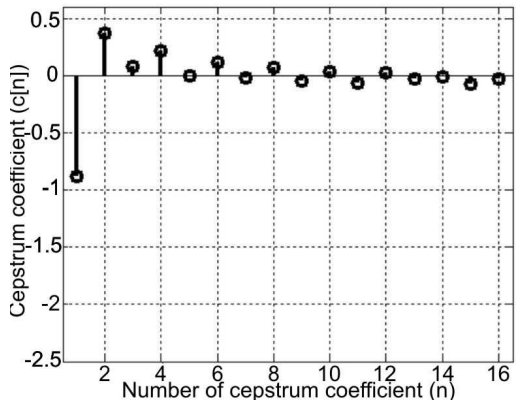

a)

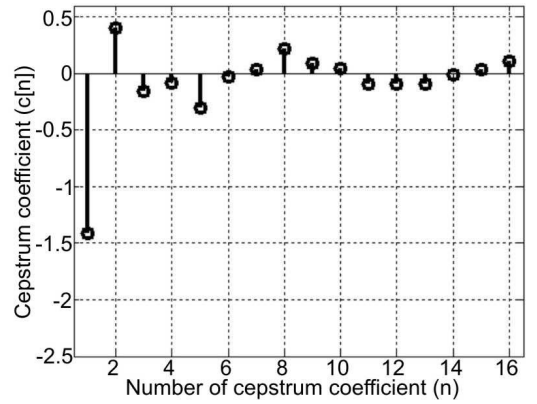

b)

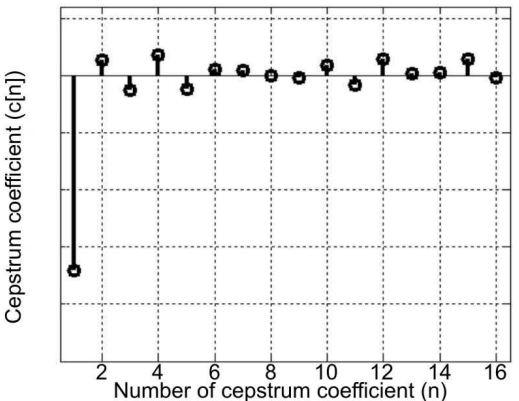

c)

Fig. 4. Real cepstrum coefficients of radar echoes target samples; a) person walking, b) group of persons running, c) vehicle 
In Fig. 5 are presented projections of the second and the third real cepstrum coefficients for three analysed classes and for different sequence durations.

From Figs. 5a and b can be concluded that there is no significant difference in the second and third real cepstrum coefficients values for 1 and $2 \mathrm{~s}$ sequence durations. In these values information about central Doppler frequency is kept, which is one of the major characteristics for classification.

Furthermore, as the sequences duration decrease, the second and the third real cepstrum coefficients from various classes are closer (see Fig. 5c). This can be explained with the fact that there is no enough information for proper separation of classes.

In the second experiment we used 20 sequences from each of three major group of moving targets (one person, group of persons and vehicle). All these sequences were grouped in two classes based on spectral width: below $40 \mathrm{~Hz}$ and above $100 \mathrm{~Hz}$. Sequence duration from 0.125 to $2 \mathrm{~s}$, too. The same number of points used to calculate the discrete Fourier transform as the first experiment.

In Fig. 6 projections of the fifth and the sixth real cepstrum coefficients for two analysed classes and for different sequence durations are presented.

From Figs. 6a and $\mathrm{b}$ it can be concluded that there is no significant difference in the fifth and sixth real cepstrum coefficients values for 1 and $2 \mathrm{~s}$ sequence durations. In these values is information about spectral width around central Doppler frequency is kept, which is one of the major characteristics for classification.

Furthermore, as the sequences duration decrease, the fifth and the sixth real cepstrum coefficients from various classes are closer (see Fig. 6c). This can be explained with the fact that there is not enough information for proper separation of classes.

\section{CONCLUSION}

In this paper the database of radar echoes from various targets has been described. The database is available for public download.

The spectral analysis conducted in this paper is used to extract very basic information that could be used for classification. It was not intended to identify individuals or classes of people from their gait. More sophisticated techniques would be needed to resolve the contributions to the gait motion from body parts like the arms, upper leg, lower leg, and foot.

We believe that such a publicly available database will allow easier comparison and performance evaluation of the existing and future classification algorithms. a)

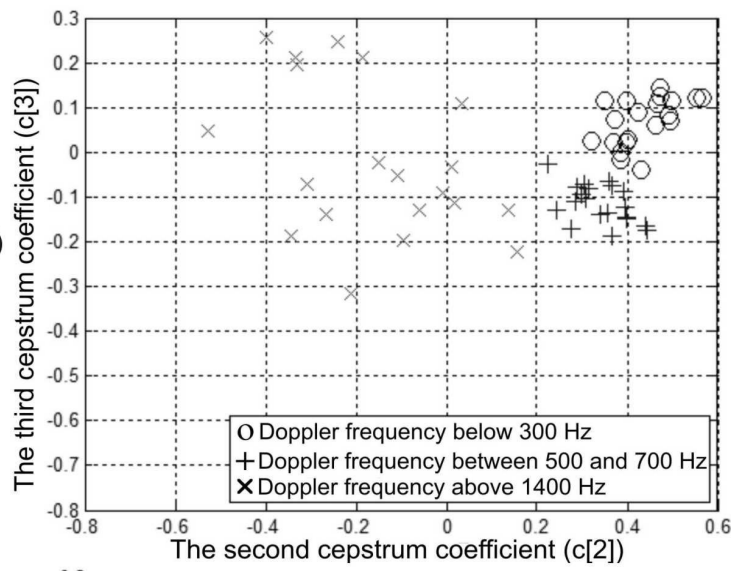

b)

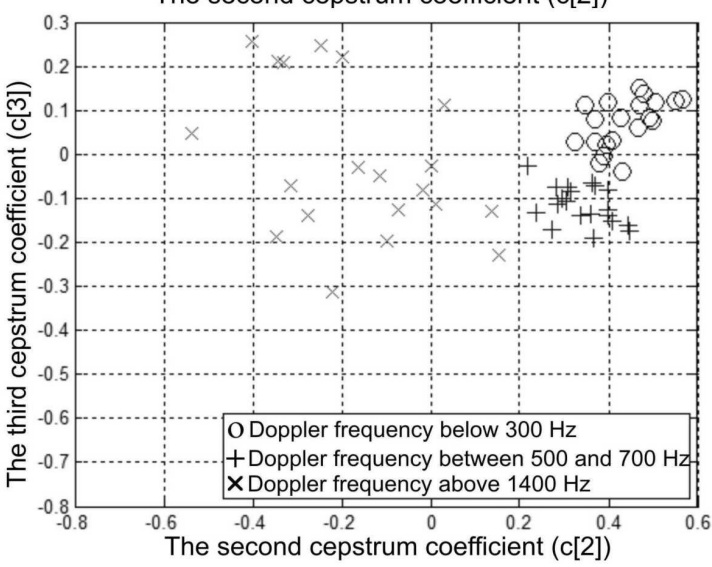

c)

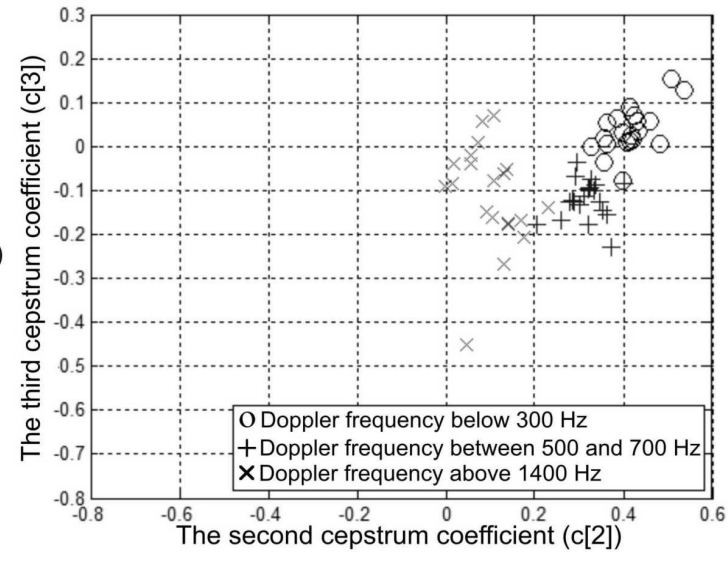

Fig. 5. The second and the third real cepstrum coefficients for different sequence duration; a) $2 \mathrm{~s}$, b) $1 \mathrm{~s}$, c) $0.125 \mathrm{~s}$

In future work we will extend our database with new target classes (tanks, helicopters, animals). We will also provide a database for target detection and classification research.

Also, we developed and analyzed cepstrum coefficients of the real audio (acoustic) radar signals. It has been showed that the second and the third cepstrum coefficients give promising information 
a)

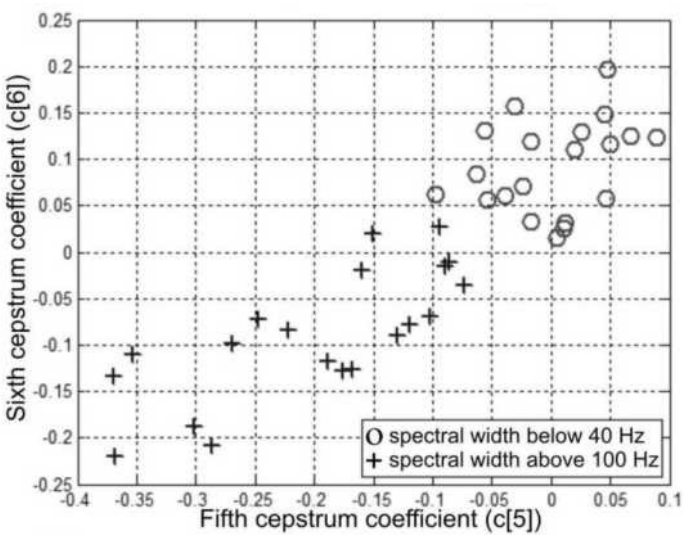

b)

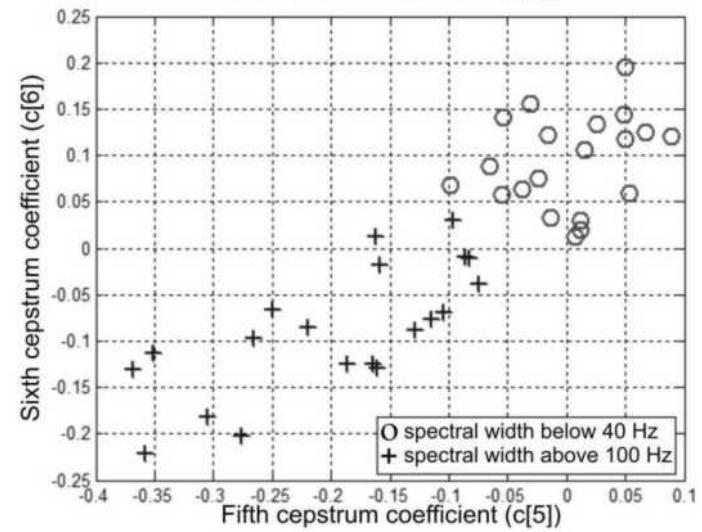

c)

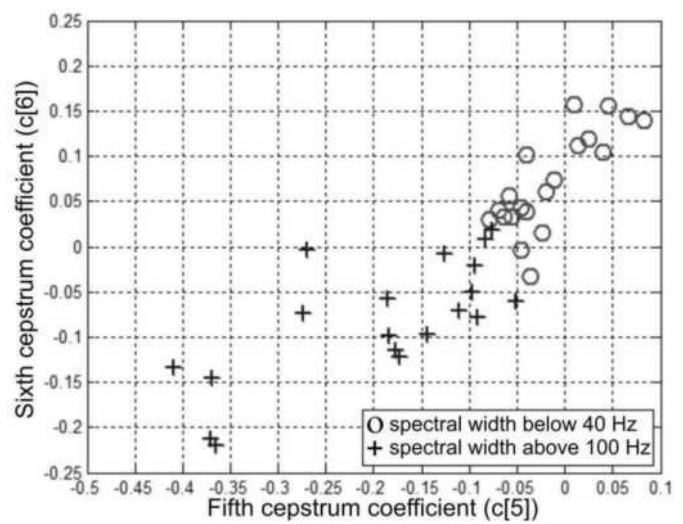

Fig. 6. The fifth and the sixth real cepstrum coefficients for different sequence duration; a) $2 \mathrm{~s}$, b) $1 \mathrm{~s}$, c) $0.125 \mathrm{~s}$

about central Doppler frequency, while the fifth and the sixth cepstrum coefficients give promising information about the spectral width around it. The cepstrum-based analysis conducted in this paper is used to extract very basic information that could be used for target types estimations. In future work we will use these notices to develop cepstrum-based classification algorithms and to develop further job for automatic recognizing software and hardware.

\section{ACKNOWLEDGMENT}

Results presented in this paper are made within project No 47029 financed by the Ministry of Science and Technology Development, the Republic of Serbia.

\section{REFERENCES}

[1] Virtić, M. P., Aberšek, B., Župerl, U. (2008). Using of Acoustic Models in Mechanical Diagnostics. Strojniški vestnik - Journal of Mechanical Engineering, vol. 54, no. 12 , p. 874-882.

[2] Solodov, I., Doring, D., Busse, G. (2011). New opportunities for NDT using non-linear interaction of elastic waves with defects. Strojniški vestnik - Journal of Mechanical Engineering, vol. 57, no. 3, p. 169-182.

[3] Avci, E., Turkoglu, I., Poyraz, M. (2006). A new approach based on wavelet nero genetic network for automatic target recognition with X-band Doppler radar. Journal of Electrical \& Electronics Engineering, vol. 6, no. 2, p. 157-168.

[4] Bilik, I., Tabrikian, J. (2007). Knowledge-based target classification for Doppler radars in knowledge-based radar detection. Gini, F., Rangaswamy, M. (eds.) Tracking, and Classification, John Wiley \& Sons, Hoboken, ch. 9, p. 197-224

[5] Geisheimer, J.L., Marshall, W.S., Greneker, E. (2001). A continuous-wave radar for gait analysis. 35th Asilomar Conference on Signals, Systems, and Computers, Conference Proceedings, vol. 1, p. 834838.

[6] van Dorp, P., Groen, F.C.A. (2003). Human walking estimation with radar. IEE Radar Sonar Navigation, Proceedings, vol. 150, no. 5, p. 356-365.

[7] Otero, M. (2005). Application of a continuous wave radar for human gait recognition. Proceedings of SPIE: Signal Processing, Sensor Fusion, and Target Recognition XIV, vol. 5809, p. 538-548.

[8] Greneker, G. (2005). Very low cost stand-off suicide bomber detection system using human gait analysis to screen potential bomb carrying individuals. Proceedings of SPIE: Radar Sensor Technology IX, vol. 5788, p. 46-56.

[9] Thayaparan, T., Abrol, S., Riseborough, E., Stankovic, Lj., Lamothe, D., Duff, G., (2007). Analysis of radar micro-Doppler signatures from experimental helicopter and human data. IET Radar Sonar Navigation, vol. 1, no. 4, p. 289-299, DOI:10.1049/iet-rsn:20060103.

[10] Jahangir, M., Ponting, K.M., O’Loghlen, J.W. (2003). Robust Doppler classification technique based on hidden Markov models. IEEE Proceeding-Radar Sonar Navigation, vol. 150, no. 1, p. 33-36, DOI:10.1049/iprsn:20030027.

[11] Andrić, M., Đurović, Ž., Zrnić, B. (2005). Ground surveillance radar target classification based on fuzzy logic approach. Proceedings of International Conference on Computer as a Tool, vol. 2, p. 13901392. 
[12] Bilik, I., Tabrikian, J., Cohen, A. (2006). GMM-based target classification for ground surveillance Doppler radar. IEEE Transaction of Aerospace and Electronic Systems, vol. 42, no. 1, p. 267-278, DOI:10.1109/ TAES.2006.1603422.

[13] Bilik, I., Tabrikian, J. (2007). Radar target classification using Doppler signatures of human locomotion models. IEEE Transaction of Aerospace and Electronic Systems, vol. 43, no. 5, p. 1510-1522, DOI:10.1109/ TAES.2007.4407474.
[14] The database of radar echoes from various targets, from: http://cid-3aaf3e18829259c0.skydrive.live.com/ home.aspx, accessed on 10-04-21.

[15] McConaghy, T., Leung, H., Bosse, E., Varadan, V. (2003). Classification of audio radar signals using radial basis function neural networks. IEEE Transactions on Instumentation and Measurement, vol. 52, no. 6, p. 1771-1779, DOI:10.1109/TIM.2003.820450. 